\title{
LINTERPRETAZIONE ADEGUATRICE TRA TRIBUNALE COSTITUZIONALE E GIUDICI COMUNI IN SPAGNA ${ }^{1}$
}

\author{
GiANCARLO Rolla \\ Catedrático de Derecho Constitucional. \\ Departamento de Derecho Público y Procesal. \\ (Área de Derecho Constitucional). \\ Facultad de Derecho, Universidad de Génova \\ Rolla@unige.it>
}

SOMMARIO: 1 . I sistemi di giustizia costituzionale e la loro incidenza sull'autonomia interpretativa dei giudici. 2. La natura e i limiti del potere interpretativo dei giudici comuni. 3. La posizione istituzionale del Tribunale costituzionale e la problematica distinzione tra controllo di legalità ordinaria e costituzionale. 4. L'interpretazione conforme a Costituzione secondo la dottrina del Tribunale costituzionale. 5. La forza giuridica della giurisprudenza del Tribunale costituzionale e le sentenze intepretative. 6. L'interpretazione conforme a Costituzione nei ricorsi di amparo costituzionale.

\section{I SISTEMI DI GIUSTIZIA COSTITUZIONALE E LA LORO INCIDENZA SULL'AUTONOMIA INTERPRETATIVA DEI GIUDICI}

Nell'introdurre il tema dell'interpretazione conforme a Costituzione e delle conseguenti relazioni fra Tribunale costituzionale e giudici comuni in Spagna vorrei prendere le mosse da una considerazione preliminare ovvia, ma utile per comprendere i temi specifici e le tensioni che hanno interessato l'ordinamento costituzionale spagnolo: che, cioè, questa problematica -comune a tutti gli ordinamenti che si ispirano al costituzionalismo- assume connotati differenti in ragione tanto del tipo di sistema di giustizia costituzionale adottato, quanto delle diverse competenze attribuite al giudice costituzionale.

E' evidente che il tema dell'interpretazione conforme a Costituzione è parte costitutiva del controllo di costituzionalità, in quanto si collega direttamente ad una delle sue funzioni essenziali: non a caso, il Tribunale costituzionale spagnolo ha ripetutamente legato questa tecnica di interpretazione al principio di supremazia

\footnotetext{
${ }^{1}$ Enviado por su autor el 16 de septiembre de 2010, para su publicación en Estudios Constitucionales.
} 
della Costituzione di cui all'art. 9 della Costituzione spagnola (CE). Compete, d'altra parte, alla giustizia costituzionale favorire la coerenza del sistema giuridico: non solo espungendo le possibili antinomie interne all'ordinamento -costituite dalla presenza di norme di legge contrastanti con quelle di rango costituzionale-, ma anche favorendo un'interpretazione delle norme legali coerenti con le disposizioni della Costituzione.

In alcuni ordinamenti tale competenza è espressamente codificata-come nel caso del Tribunale costituzionale della Repubblica federale tedesca o del Tribunale costituzionale spagnolo-; in altri sistemi, sia pure in assenza di esplicite previsioni normative, si perviene ai medesimi risultati in via di fatto, a causa dell'esistenza del principio dello stare decisis o in conseguenza dell'auctoritas di cui godono le sentenze delle Corti costituzionali, della loro forza persuasiva, del consenso che le stesse acquisiscono presso gli operatori giuridici e gli interpreti del diritto.

$\mathrm{Va}$, tuttavia, considerato che le problematiche connesse all'intepretazione conforme a Costituzione si manifestano in modo e in misura sostanzialmente differenti negli ordinamenti di common law a Costituzione rigida -ove opera la judicial review of legislation- rispetto a quelli ove si è optato per un sistema di giustizia costituzionale accentrato, esercitato da un organo esterno all'ordine giudiziario.

Nei primi, infatti, la necessità di ricercare l'interpretazione conforme a Costituzione è parte integrante del controllo di legalità e rappresenta uno dei canoni di interpretazione che i giudici debbono utilizzare; d'altra parte, tale attività ermeneutica può essere considerata espressione di un'intepretazione logico-sistematica, in base alla quale la norma legale fa sistema con quella costituzionale. In altri termini, il giudice deve fisiologicamente decidere se applicare una disposizione attribuendole un significato conforme a Costituzione ovvero disapplicarla, essendo possibile ricavare dalla disposizione significati conformi a Costituzione.

La problematica dell'interpretazione conforme a Costituzione si pone, invece, in modo differente negli ordinamenti ove operano sistemi astratti ed accentrati di giustizia costituzionale: in questo caso il controllo di costituzionalità è separato da quello di legalità e riservato in via esclusiva ad un potere esterno a quello giudiziario. Cosicchè l'autonomia interpretativa del giudice (che comprende anche la ricerca di un'intepretazione conforme a Costituzione delle disposizioni che deve applicare) si deve confrontare e contemperare con il monopolio interpretativo dei Tribunali costituzionali per quanto concerne la conformità delle norme alla Costituzione. $^{2}$

\footnotetext{
${ }^{2}$ Si veda, sia pure con riferimento specifico alla realtà italiana: R. Romboli, Linterpretazione della legge alla luce della Costituzione tra Corte costituzionale e giudice comune, in Il dialogo tra le Corti, Pisa, 2004, 25 ss.
} 
In questo caso, la difficoltà nel tracciare una linea di demarcazione tra le due funzioni è intrinseca alla natura del sistema adottato: si può parlare, in proposito, secondo il pensiero di un illustre giurista spagnolo di debilidades inherentes al modello kelseniano. ${ }^{3}$ Inoltre, essa è accentuata in quei sistemi in cui, per un verso, la natura accentrata del controllo di costituzionalità è incrinata dall'attribuzione ai giudici comuni di un sostanziale potere di disapplicare le norme contrarie a fonti di rango superiore -come nel caso della normativa comunitaria e convenzionale in materia di diritti fondamentali-: mentre, per un altro, verso il carattere astratto del giudizio è attenuato sia dal tipo di competenze attribuite al Tribunale costituzionale -come nel caso dei ricorsi diretti a tutela dei diritti fondamentali- sia dai modi di accesso alla giustizia costituzionale previsti nei singoli ordinamenti -come, ad esempio, nelle questioni di incostituzionalità-.

Quest'ultima sottolineatura induce a considerare che l'incidenza dell'interpretazione dei Tribunali costituzionali sull'autonomia interpretativa dei giudici comuni si modula in modi differenti a secondo della competenza che il giudice costituzionale esercita.

E' evidente che il controllo di legalità ordinaria e quello di costituzionalità si pongono su piani distinti e non sono suscettibili di entrare in conflitto in tutti i casi in cui il giudice costituzionale esercita un controllo di tipo preventivo -sia di natura generale, sia in relazione a specifiche competenze come il controllo sulla legittimità dei trattati internazionali in Spagna o sugli Statuti delle Regioni ordinarie in Italia-.

Si tratta, tuttavia, di situazioni circoscritte, mentre nella maggior parte dei casi tra la giurisdizione costituzionale e quella comune, si instaura una relazione all'interno della quale si annidano spesso i germi di effetti perturbatori l'ordinario fluire dell'intepretazione e dell'applicazione del diritto: un intreccio che -come si vedrà in seguito- è assai forte nei giudizi sui ricorsi diretti a tutela dei diritti fondamentali, meno problematico nelle questioni di costituzionalità. ${ }^{4}$

\footnotetext{
${ }^{3}$ Così: F. Rubio Llorente, "Divide et obtempera? Una reflexión desde España sobre el modelo europeo de convergencia de jurisdicciones en la protección de los derechos", in Revista española de derecho constitucional, 2003, 53.

${ }^{4}$ Sulla problematica generale dei rapporti tra giurisdizione costituzionale e comune in Spagna si veda: A. DE Oliva SANTOS, "Tribunal constitucional y jurisdicción ordinaria: causas, ámbitos y alivios de una tensión”, in Tribunal constitucional, jurisdicción ordinaria y derechos fundamentales, Madrid, 1996, 3 ss.; L. LÓPEZ GUERRA, Jurisdicción ordinaria y jurisdicción constitucional, in La aplicación jurisdiccional de la Constitución, Valencia, 1997, 29 ss.; S. Ortiz Herrera, "Articulación del Tribunal constitucional y del poder judicial en la labor interpretativa de la Constitución. Especial referencia a las sentencias interpretativas del Tribunal constitucional", in Boletín de la Facultad de Derecho, UNED, 1997, 633 ss.; P. Pérez Tremps, Tribunal Constitucional y Poder judicial, Madrid, 1985; F. Rubio Llorente, "Sobre la relación entre Tribunal constitucional y poder judicial en el ejercicio de la jurisdicción constitucional”, in La forma del poder (estudios sobre la Constitución),
} 
Se queste considerazioni preliminari possono introdurre la problematica generale dei rapporti tra giudice costituzionale e giudici comuni nei sistemi di giustizia costituzionale di ispirazione kelseniana, va -tuttavia- precisato che ciascun ordinamento presenta a sua volta delle specificità. E queste, nel caso dell'ordinamento spagnolo, sono costituite essenzialmente: a) dalla diversità del dato normativo -di rango costituzionale e subcostituzionale- di riferimento; b) dalla rilevanza assorbente che esercita la competenza del Tribunale costituzionale in materia di ricorsi di amparo costituzionale; c) da una maggiore difficoltà a stemperare le occasioni di conflittualità ed a trovare un dialogo tra il Tribunale supremo e il Tribunale costituzionale.

\section{LA NATURA E I LIMITI DEL POTERE INTERPRETATIVO DEI GIUDICI COMUNI}

L'ordinamento spagnolo, a differenza di quello italiano, dedica diverse disposizioni -soprattutto della Legge organica sul potere giudiziario (LOPJ) e della Legge organica sul Tribunale costituzionale (LOTC) - ai rapporti tra potestà interpretativa dei giudici e valore della giurisprudenza costituzionale, nel tentativo di regolare entro un alveo prestabilito il flusso delle relazioni tra il Tribunale costituzionale ed i giudici comuni. L'esperienza, tuttavia, ha insegnato che tale risultato non è stato del tutto conseguito, in quanto le occasioni di conflitto sono risultate tutt'altro che marginali, sino al punto di rischiare -come è stato affermato- di porre in pericolo l'intero sistema di relazioni tra i due poteri. ${ }^{5}$

In particolare, a nostro avviso, la normativa si premura di affrontare alcuni profili della materia inerenti alla natura e ai limiti del potere interpretativo dei giudici comuni, ad una possibile ripartizione di competenza tra le due giurisdizioni, alla forza giuridica della giurisprudenza del Tribunale costituzionale. ${ }^{6}$

In ordine al primo aspetto, si può richiamare -innanzitutto- l'art. 6.1. del Codice civile che annovera l'intepretazione giurisprudenziale tra le fonti legali del diritto, precisando che la " jurisprudencia completará el ordenamiento jurídico”.

Madrid, 1993, 463 ss.; C. STARCK, "Jurisdicción constitucional y Tribunales ordinarios", in Revista española de derecho constitucional, 1998, 11 ss.

${ }^{5}$ Per quanto concerne alcuni dei modi attraverso i quali si manifesta la c.d. "guerra tra le due Corti" si vedano i lavori di: R. SERRA CRISTÓbAL, La guerra de las Cortes: la revisión de la jurisprudencia del Tribunal supremo a través del recurso de amparo, Madrid, 1999; C. Aguado Renedo, "Del intento de control del Tribunal constitucional por la Sala de lo civil del Tribunal supremo o de cómo poner en riesgo todo un sistema", in Revista general derecho constitucional, 1, 2006; E. Delgado del Rincón, "Los conflictos entre la Sala primera del Tribunal supremo y el Tribunal constitucional: génesis, evolución y algunas propuestas de solución”, in Revista general derecho constitucional, 4, 2007.

${ }^{6}$ Cfr., R. PUnSET, J. SANTOS (dirs.), Jurisdicción ordinaria y Tribunal constitucional, Santiago de Compostela, 1997. 
Tale disposizione è stata, poi, interpretata nel senso che la jurisprudencia proviene da un consolidato orientamento giurisprudenziale ricavabile dalle decisioni del Tribunale supremo. ${ }^{7}$

L' art. 6 del codice civile assegna, quindi, al Tribunale supremo una particolare posizione all'interno del sistema giurisdizionale, che può essere sintetizzata nella competenza ad unificare la doctrina delle diverse giurisdizioni (penale, civile, del lavoro, amministrativa). E lo strumento per conseguire tale risultato è stato individuato nel "recurso de casación", che consente al Tribunale supremo di "revisar la aplicación de la ley tanto sostantiva como procesal hecha por el tribunal de la instancia" al fine di assicurare i principi di sicurezza giuridica e di eguale applicazione del diritto. ${ }^{8}$

Appare evidente che i rischi di sovrapposizione tra la giurisprudenza del Tribunale costituzionale e quella del Tribunale supremo siano molto forti quando i due organi non forniscono una "stessa" interpretazione conforme a Costituzione e quando il giudice costituzionale utilizza la "sua" intepretazione per risolvere un ricorso diretto a tutela dei diritti fondamentali.

Altre disposizioni rilevanti si rinvengono nel comma 1 e 3 dell'art. 5 della LOPJ.

Il primo, dopo aver affermato che la Costituzione è la fonte che si pone al vertice dell'ordinamento giuridico e vincola tutti i giudici, dispone (proprio in conseguenza di ciò) che questi "interpretarán y aplicarán las Leyes y los Reglamentos según los preceptos y principios constitucionales”. Da tale disposizione deriva l'obbligo per i giudici, per un verso, di interpretare le norme conformemente alla Costituzione e, per un altro verso, di non applicarle qualora risulti impossibile conferire loro un significato compatibile con il dettato costituzionale.

Se il principio che le leggi si debbono presumere conformi a Costituzione rappresenta una premessa del sistema, la ricerca di una possibile interpretazione compatibile con le disposizioni costituzionali costituisce un'operazione logica che compete a tutti gli operatori giuridici, ma in particolar modo ai giudici, in quanto interpreti qualificati del diritto. ${ }^{9}$ Tale acquisizione trova una conferma nel succes-

\footnotetext{
${ }^{7}$ Sulla funzione di unificazione degli orientamenti giurisprudenziali del Tribunale supremo si rinvia a: M. DEL Pilar GarCía Ruiz, "El Tribunal supremo como mero órgano unificador de doctrina: la posible afectación de su garantía institucional", in Revista española de derecho constitucional, 2008, 119 ss.

${ }^{8}$ Sul problema specifico si veda: E. CANCER LALANNE, La Constitución como motivo del recurso de casación, in Cuadernos de derecho público, 1999, 111 ss.

9 In merito al ruolo dell'intepretazione nell'applicazione delle disposizioni della Costituzione: AA.VV., La aplicación jurisdiccional de la Constitución, Valencia, 1997; M. CARrILlo, La aplicación judicial de la Constitución, in Revista española de derecho constitucional, 2004, 73 ss.; J. CARMONA TINOCO, La interpretación judicial
} 
sivo comma 3 dell'art. 5 della LOPJ, ove si precisa che il giudice può proporre la questione di incostituzionalità di una determinata disposizione soltanto "cuando por vía interpretativa no sea posible la acomodación de la norma al ordenamiento constitucional”. Di conseguenza, anche i giudici debbono utilizzare le norme costituzionali come criteri per l'interpretazione sistematica dell'ordinamento giuridico vigente e tale operazione è fisiologica a condizione che non si realizzi attraverso un percorso interpretativo irrazionale, che forzi il significato evidente dell'enunciato normativo.

In virtù di tale attività interpretativa, i giudici comuni sono parte del controllo di costituzionalità non solo perché costituiscono la principale "porta di accesso" al sindacato di costituzionalità della legge, ma anche in quanto esercitano quest'ultimo come parte del controllo di legalità.

Però, il sindacato del giudice comune e di quello costituzionale non si equivalgono. Infatti, dinanzi ad una disposizione suscettibile di interpretazioni diverse (di cui una conforme ed un'altra sicuramente contraria alla Costituzione) il giudice deve necessariamente optare per la prima, mentre il Tribunale costituzionale si trova dinanzi ad una duplice possibilità, potendo scegliere tra una sentenza adeguatrice o di accoglimento: cioè, tra conservare la disposizione affermando una sua determinata intepretazione, ovvero conservare la disposizione impedendo con effetti erga omnes l'utilizzazione di un'interpretazione contraria alla Costituzione. ${ }^{10}$

Un ulteriore elemento di differenziazione tra l'attività interpretativa dei giudici e quella del Tribunale costituzionale discende dalla proposizione che conclude l'art. 5.1 della LOPJ, secondo la quale l'interpretazione dei giudici deve risultare conforme a quella fornita dalla giurisprudenza costituzionale. Cosicché, l'attività giudiziale deve, comunque, svolgersi nell'ambito della doctrina del Tribunale costituzionale. Viceversa, il giudice costituzionale non è formalmente vincolato all'interpretazione conforme che i giudici propongono, neppure nei casi in cui tale interpretazione sia consolidata ovvero confermata dal Tribunale supremo.

Quest'ultima precisazione prospetta una questione delicata, in quanto il Tribunale costituzionale sembra non aderire del tutto alla teoria del "diritto vivente": il

de la Constitución, Madrid, 1996; J. LA Fuente BALle, La jurisdicialización de la intepretación constitucional, Madrid, 2000.

${ }^{10}$ Tale distinzione era già stata colta con la solita sottigliezza da: V. CRISAFulLI, "Questioni in tema di interpretazione costituzionale nei rapporti con l'interpretazione giudiziaria”, in questa Revista, 1956, 929 ss. Con riferimento alla realtà spagnola si rinvia a: S. OrTiz Herrera, Articulación del Tribunal constitucional y del poder judicial en la labor interpretativa de la Constitución. Especial referencia a las sentencias interpretativas del Tribunal constitucional, cit., 633 ss. 
che ripropone il dubbio se il controllo di costituzionalità debba avere ad oggetto la norma legale ovvero la norma così come è concretamente applicata. ${ }^{11}$

In proposito, è interessante il dibattito che si è aperto attorno alla STC 131/06, nella quale il plenum si è diviso sul punto se il controllo di costituzionalità si debba basare sulla norma legale o sulla norma "vivente" quale scaturisce dall'intepretazione conforme a Costituzione fornita dal Tribunale supremo. Sul punto, all'interno del collegio si è determinata una divaricazione: la maggioranza, precisando che, in virtù del principio di conservazione della legge, debbono essere dichiarate incostituzionali soltanto le disposizioni per le quali è impossibile conseguire un'intepretazione conforme a Costituzione, ${ }^{12}$ ha manifestato la necessità di esplorare se tra le diverse possibilità interpretative ve ne sia almeno una conforme a Costituzione. ${ }^{13}$ Per contro, tre giudici, tra i quali la Presidente, hanno ritenuto nel loro voto particolare che il procedimento argomentativo seguito dal Tribunale determinasse una rottura nella continuità di un orientamento giurisprudenziale secondo cui "a través de la cuestión, se enjuicia no la interpretación judicial de la ley, sino la ley misma, o dicho más propiamente, no la norma legal producto de la interpretación, sino la disposición normativa en sí misma considerada, esto es, el texto o proposición legal".

In particolare, i giudici dissenzienti appoggiavano la loro critica su diversi precedenti, tra i quali merita, per un verso, ricordare un'iniziale pronuncia del Tribunale costituzionale nella quale si affermava che "no compete, pues, al Tribunal, en su función de órgano decisor de los recursos de inconstitucionalidad, enjuiciar el mayor o menor acierto con que los operadores jurídicos estén llevando a cabo la labor de aplicación". ${ }^{14}$ A loro avviso, la finalità delle questioni di incostituzionalità non dovrebbe consistere nel "resolver controversias interpretativas sobre la legalidad entre órganos jurisdiccionales o dudas sobre el alcance de determinado precepto legal". Di conseguenza, sarebbe precluso al Tribunale costituzionale, in sede di controllo di costituzionalità (la situazione è diversa nel caso dei ricorsi di amparo, come si vedrà in seguito) utilizzare un'interpretazione giudiziale come criterio per rigettare una questione di incostituzionalità.

Appare evidente che, ad avviso degli autorevoli giudici che hanno formulato il voto particolare non sarebbe opportuno ricorrere a pronunce c.d. "correttive",

\footnotetext{
${ }^{11}$ Sul punto si veda: M. ARAGÓn ReYes, "La interpretación de la Constitución y el carácter objetivado del control jurisdiccional”, in Revista española de derecho constitucional, 1986, 85 ss.

${ }^{12}$ Cfr., SSTC 4/81, SSTC 111/93, STC 24/04, STC 138/05.

${ }^{13}$ Cosi, ad esempio, in: STC 76/96, STC 138/05.

${ }^{14}$ Come la STC $11 / 81$.
} 
nelle quali il giudice costituzionale non affronta tanto il merito della questione, ma si limita a rilevare che l'interpretazione data dal giudice a quo non è corretta, dal momento che non ha tenuto conto del "diritto vivente", ovvero dell'orientamento giurisprudenziale manifestato dal Tribunale supremo.

L'auspicio che -a nostro avviso- sta alla base della posizione sostenuta dai tre magistrati dissenzienti è individuabile nella necessità di ricercare dei canoni comportamentali e dei criteri intepretativi in grado di tracciare, in sede di esame delle questioni di incostituzionalità, una demarcazione tra le rispettive sfere di autonomia.

E questo è un profilo problematico della materia che la legislazione organica ha tentato di affrontare, sia pure con esiti non risolutivi. ${ }^{15}$

\section{LA POSIZIONE ISTITUZiONALE DEL TRIBUNALE COSTITUZIONALE \\ E LA PROBLEMATICA DISTINZIONE TRA CONTROLLO \\ DI LEGALITÀ ORDINARIA E COSTITUZIONALE}

Tanto nella Costituzione, quanto nella LOTC si rinvengono disposizioni finalizzate a regolare la particolare posizione istituzionale che occupa il Tribunale costituzionale. Se, da un lato, si può richiamare l'art. $117 \mathrm{CE}$, il cui comma terzo precisa che l'esercizio della funzione giurisdizionali in ogni tipo di procedimento è di esclusiva competenza dei giudici predeterminati dalla legge, secondo le competenze e i procedimenti dalla stesse stabilite; dall'altro lato, secondo la disposizione dell'art. 161.1 CE tutti i pubblici poteri sono soggetti alle decisioni del giudice costituzionale. Mentre, l'art. 40.2 della LOTC rafforza tale principio, precisando che "en todo caso, la jurisprudencia de los tribunales de justicia recajdas sobre leyes, disposiciones y actos enjuiciados por el TC habrà de entenderse corregida por la doctrina derivata de las sentencias y autos que resulevan los processos constitucionales". Inoltre, l'art. $123 \mathrm{CE}$, dopo avere qualificato il Tribunale supremo come "l'organo jurisdiccional superior en todos los ordines", precisa "salvo lo dispuesto en materia de garantías constitucionales".

La posizione istituzionale del giudice costituzionale è, poi, rafforzata dall'art. 4 della LOTC, il quale nella sua formulazione originaria disponeva che in nessun caso si potrà promuovere questione di competenza o di giurisdizione da parte del

\footnotetext{
${ }^{15}$ Si veda: A. De Oliva Santos, "Tribunal constitucional y jurisdicción ordinaria: causas, ámbitos y alivios de una tensión", in Tribunal constitucional, jurisdicción ordinaria y derechos fundamentales, Madrid, 1996, 3 ss.; L. López Guerra, "Jurisdicción ordinaria y jurisdicción constitucional", in La aplicación jurisdiccional de la Constitución, cit., 1997, 29 ss.

Per un giudizio generale sulla legge di riforma della LOTC: M. ARAGÓn ReYeS, "La reforma de la Ley Orgánica del Tribunal constitucional”, in Revista española de derecho constitucional, 2009, 11 ss.
} 
Tribunale costituzionale e che compete a tale organo valutare la propria eventuale carenza di giurisdizione o di competenza. ${ }^{16}$ Siffatta disposizione è stata successivamente integrata dalla legge organica n. 6/2007 (di riforma della LOTC), che ha introdotto un'ulteriore disposizione, con l'obiettivo di proteggere il Tribunale costituzionale da possibili intromissioni nel proprio territorio di competenza: in base ad essa compete al Tribunale costituzionale delimitare -di ufficio o su istanza di parte- il proprio ambito di giurisdizione, potendo a tal fine adottare tutti gli strumenti necessari per preservarla "incluyendo la declaración de nulidad de aquellos actos o resoluciones que la menoscaben". ${ }^{17}$

La disposizione appena richiamata -chiaramente influenzata dalla grave crisi istituzionale intercorsa tra i due organi supremi, che si culminò nella sentenza della Sala primera del TS del 23 gennaio 2004 che ha dichiarato la responsabilità civile di 11 dei 12 magistrati costituzionali, con la motivazione che non decidendo nel merito un ricorso (ma dichiarandolo inammissibile), aveva leso il diritto ad una tutela giudiziale effettiva del ricorrente- ${ }^{18}$ ribadisce sia la supremazia del Tribunale costituzionale in materia costituzionale, sia l'impossibilità di sanzionare il Tribunale costituzionale, qualora eventualmente esorbiti dalle proprie competenze.

La posizione istituzionale che tanto la Costituzione, quanto la LOTC riconoscono al giudice costituzionale apre, tuttavia, la porta a sue possibili "incursioni" in materia di controllo di legalità ordinaria: sia in quanto il soprarichiamato art. 4 della LOTC fa del Tribunale costituzionale l'arbitro insindacabile della propria competenza, sia perché il significato normativo da attribuire alle disposizioni di legge è parte necessaria del giudizio costituzionale. Già Crisafulli, all'inizio dell'esperienza italiana di giustizia costituzionale, evidenziava che l'accertamento del vizio di legittimità costituzionale implica "necessariamente l'interpretazione

\footnotetext{
${ }^{16}$ A proposito dell'autonomia del Tribunale costituzionale nell'esercizio delle proprie competenze si rinvia a: P. Rodríguez Patrón, La "autonomía procesal" del Tribunal constitucional, Madrid, 2003.

${ }^{17}$ La riforma della Legge organica sul Tribunale costituzionale è stata oggetto di numerosi commenti dottrinali. Tra questi, con particolare riferimento alla problemática sollevata nel testo, si segnalano: F. BALAGUER Callejón, La nueva ley orgánica del Tribunal constitucional, Madrid, 2008; M. CARILlo, "La reforma de la jurisdicción constitucional: la necesaria racionalización de un órgano constitucional en crisis", in Hacia una nueva jurisdicción constitucional, Valencia 2008, 70 ss.; S. GARCíA VÁZQUEZ, La controvertida reforma de la Ley Orgánica del Tribunal constitucional, Santiago de Compostela, 2009; P. Pérez Tremps (coord.), La Reforma del Tribunal constitucional, Valencia, 2007.

${ }^{18}$ Per una ricostruzione della vicenda si rinvia a: E. Delgado DEL RinCÓn, Los conflictos entre la Sala primera del Tribunal supremo y el Tribunal constitucional: génesis, evolución y algunas propuestas de solución, cit., 4, 2007. Per una prospettiva più ampia si veda: J. L. Manzanares Samaniego, "La delimitación de competencia entre el Tribunal Supremo y el Tribunal Constitucional", in La aplicación jurisdiccional de la Constitución, cit., 63 ss.
} 
della norma di grado superiore, che si assume violata, ed eventualmente delle norme di legge ordinaria, cui dovrebbe darsi applicazione". ${ }^{19}$

Pertanto, tracciare una linea di demarcazione netta tra controllo di legalità e di costituzionalità risulta impossibile, dal momento che, in tutti gli ordinamenti che hanno optato per un sistema astratto e accentrato di giustizia costituzionale, l'esperienza concreta ha contraddetto la semplicità della formula secondo la quale la Corte costituzionale sarebbe "la vestale della Costituzione e la magistratura la vestale della legge". ${ }^{20}$ Inoltre, la possibilità per il Tribunale costituzionale di trovare un canone interpretativo delle norme primarie in grado di preservare la sfera di autonomia della giurisdizione comune è soggetta a diverse variabili.

Una di queste è costituita dal tipo di competenza esercitata dal giudice costituzionale. Se le relazioni tra giurisdizione comune e costituzionale non presentano criticità nei sistemi in cui il controllo di costituzionalità è di tipo preventivo ovvero nei casi in cui il giudizio del Tribunale è attivato da un ricorso che deve essere presentato entro poco tempo dalla pubblicazione di una legge, diversa si presenta la situazione in altre situazioni che un autorevole giurista ha qualificato come sistemi orizzontali e verticali. ${ }^{21}$

I primi -tra i quali rientrano le questioni di costituzionalità- si basano sulla collaborazione tra due poteri asimmetrici: l'uno concentrato (quello costituzionale), l'altro diffuso (quello giudiziario). Da questa asimmetria scaturiscono degli effetti potenzialmente perturbatori, che riguardano tanto la dialettica tra giudice a quo e Tribunale costituzionale, quanto il dialogo tra la doctrina ricavabile dalla giurisprudenza costituzionale e gli orientamenti interpretativi della giurisdizione comune ovvero la doctrina del Tribunale supremo.

Non mancano nella giurisprudenza del giudice costituzionale spagnolo argomenti che lasciano aperta la porta a possibili incursioni nell'ambito del controllo di legalità ordinaria: ad esempio, allorchè il Tribunale costituzionale, dopo aver riconosciuto che l'interpretazione delle leggi compete al giudice ordinario, precisa che, comunque, il giudizio che compete al Tribunale costituzionale richiede "la previa consideración de una ley"; ${ }^{22}$ ovvero quando sostiene che la violazione di un diritto può essere originata non solo dall'applicazione di una legge incostituzionale, ma anche dall'applicazione erronea di una norma, per cui il giudice costituzionale

${ }^{19}$ Così in: V. Crisafulli, Lezioni di diritto costituzionale, Padova, 1984, 400.

${ }^{20}$ Così, E. De Nicola, "Discorso di insediamento alla Corte costituzionale", in questa Rivista, 1956, 169.

${ }^{21}$ Cfr., F. Rubio Llorente, Divide et obtempera? Una reflexión desde España sobre el modelo europeo de convergencia de jurisdicciones en la protección de los derechos, cit., 53.

${ }^{22}$ Cfr., STC 14/82. 
potrebbe valutare non solo se una norma è applicabile al caso concreto, ma anche se tale norma è applicata in modo erroneo. ${ }^{23}$

D'altra parte, nei sistemi di giustizia costituzionale di derivazione europea, le sentenze sono un veicolo per la circolazione della giurisprudenza e nelle decisioni del giudice costituzionale si forma tanto una dottrina costituzionale, quanto una dottrina legale. ${ }^{24}$

Non sono irrilevanti i casi in cui l' intepretazione conforme del giudice costituzionale si sovrappone a quella dei giudici comuni. Ad esempio, in alcuni casi il Tribunale costituzionale ha ammesso la propria competenza a individuare l'interpretazione adeguata a Costituzione, anche in difformità dall'interpretazione della norma legale fornita dal giudice. ${ }^{25}$ Così come in altra sentenza ha sostenuto che l'unità dell'ordinamento non tollera che i due ambiti possano essere considerati separati e incomunicabili: ${ }^{26}$ cosicchè nè la giurisdizione ordinaria -dovendo applicare una norma legale- può dimenticare l'esistenza della Costituzione, nè la giurisprudenza costituzionale può sfuggire ad un'analisi critica dell'interpretazione giudiziale.

Per contro, i sistemi "verticali" -tra i quali spiccano i ricorsi di amparo costituzionale ${ }^{27}$ escludono ogni ipotesi di collaborazione e si fondano sulla preminenza della giustizia costituzionale, la quale ha la capacità di sindacare gli atti o le omissioni dei giudici e può revisionare la loro giurisprudenza in caso di intepretazione non conforme a Costituzione.

In questo caso la tensione tra le due giurisdizioni non solo è intrinsecamente forte, ma anche difficilmente componibile: infatti, l'oggetto del giudizio del Tribunale costituzionale è costituito sia dall'applicazione giudiziale di una norma,

\footnotetext{
${ }^{23}$ Cfr., STC 46/82.

${ }^{24}$ In generale, si veda: A. De Oliva SANTOS, Tribunal constitucional y jurisdicción ordinaria: causas, ámbitos y alivios de una tensión, cit., 3 ss.; H. LÓPEz BofILL, Decisiones interpretativas en el control de constitucionalidad de la ley, Valencia, 2004; J. Santos Vijande, Doctrina y jurisprudencia del Tribunal constitucional. Su eficacia y respecto de los tribunales ordinarios, Granada, 1995.

${ }^{25}$ Cfr., STC 83/83, STC 37/86.

${ }^{26}$ Cfr., STC 50/84.

${ }^{27}$ Per una ricostruzione della disciplina e delle caratteristiche del recurso de amparo in Spagna si rinvia ai lavori di: J. Oliver Araujo, El recurso de amparo, Palma de Mallorca, 1986; M. SÁnchez Morón, El recurso de amparo constitucional, Madrid, 1987; P. CruZ Villalón, Los procesos constitucionales, Madrid, 1992; J. García Morillo, La protección judicial de los derechos fundamentales, Valencia, 1994; G. Farreres, El recurso de amparo según la jurisprudencia constitucional, Madrid, 1995; M. CARRASCO DURÁN, Los procesos para la tutela judicial de los derechos fundamentales, Madrid, 2002; E. CARMONA CUENCA, La crisis del recurso de amparo: la protección de los derechos fundamentales entre el Poder judicial y el Tribunal constitucional, Alcalá, 2005; R. Tur Ausina, Garantía de derechos y jurisdicción constitucional, Valencia, 2008.
} 
sia da una riconsiderazione dei fatti che stavano alla base del giudizio comune, cosicché tenere separati gli ambiti delle rispettive giurisdizioni risulta sostanzialmente impossibile. ${ }^{28}$

Una seconda variabile suscettibile di incidere sulle relazioni tra giurisdizione costituzionale e comune è rappresentata dalla natura della fonte che ha prodotto la norma sulla quale il Tribunale costituzionale esercita la propria attività interpretativa.

Vi sono, infatti, fattispecie con riferimento alle quali l'interpretazione della legalità ordinaria da parte del Tribunale costituzionale appare fisiologica e coerente con il sistema di giustizia costituzionale. Ad esempio, non si può negare al Tribunale costituzionale la competenza a determinare il significato costituzionale delle norme di legge che fungono da parametro per il giudizio, facendo parte -al pari delle disposizioni costituzionali- del "blocco di costituzionalità"; ovvero a valutare la corretta applicazione da parte dei giudici comuni di alcune leggi che disciplinano l'esercizio dei diritti fondamentali, al fine di valutare se si produce una lesione dell' art. 53.1 CE -il quale vieta che si produca una lesione del loro contenuto essenziale-.

Si è in presenza, infatti, di fattispecie nelle quali il controllo di legalità è strumentale alla stessa possibilità di esercitare quello di costituzionalità.

Infine, un'ulteriore variabile è costituita dai differenti modi e dalle diverse fasi in cui l'intepretazione del Tribunale costituzionale entra in relazione con il potere interpretativo dei giudici comuni. In concreto, mi pare che le relazioni tra le due giurisdizioni si pongano in termini differenti a seconda che riguardino il rapporto tra l'interpretazione del giudice costituzionale e: a) il giudice a quo, b) i giudici che dovranno applicare in futuro la giurisprudenza costituzionale, c) i giudici che in precedenza avevano fornito una determinata interpretazione delle disposizioni oggetto del giudizio di legittimità costituzionale.

Nel primo caso, il rapporto di pregiudizialità che intercorre tra il dubbio di incostituzionalità di una disposizione prospettato dal giudice e la possibilità di

\footnotetext{
${ }^{28}$ La delicatezza del potere del Tribunale costituzionale di revisionare la giurisprudenza dei giudici comuni e, in particolare, quella del Tribunale supremo è stata ampiamente avvertita dalla dottrina. In proposito si veda: L. Martín Retortillo, Del control de la discrecionalidad administrativa al control de la discrecionalidad judicial, in Revista de Administracción púbica, 1983, 1088 ss.; M. ARAGÓn REYES, "Dos cuestiones interesantes en nuestra jurisdiccion constitucional: control de las leyes anteriores y de la jurisprudencia", in El Tribunal Constitucional, Madrid, 1991, 570 ss.; R. SERRA CRISTÓBAL, La guerra de las Cortes: La revisión de la jurisprudencia del Tribunal supremo a través del recurso de amparo, Madrid, 1999; M. AlONSO IBÁNEZ, Extensiones y límites del control por el Tribunal constitucional de las resoluciones judiciales que afecten a los derechos fundamentales, Madrid, 2007; I. Díez Picazo, Reflexiones sobre el contenido y efectos de las sentencias dictadas por el Tribunal constitucional en recursos de amparo, La sentencia de amparo constitucional, Madrid, 1999, 72 ss.
} 
dare ad essa applicazione per risolvere la controversia limita indubbiamente la discrezionalità del giudice $a$ quo: al quale è sicuramente inibito di interpretare la disposizione nel significato non conforme a Costituzione e risulta vincolato all'interpretazione conforme fornita dal giudice costituzionale qualora non ne siano possibili altre. Si è, in altri termini, in presenza -per utilizzare la distinzione introdotta da un autorevole giurista- di un vincolo negativo assoluto e di un vincolo positivo relativo. ${ }^{29}$

A sua volta, il rapporto tra la doctrina del Tribunale costituzionale e l'intepretazione futura da parte dei giudici delle disposizioni che sono state oggetto di una sentenza interpretativa dovrà essere regolato -come si vedrà nel paragrafo che segue- sulla base del valore che l'ordinamento attribuisce alle sentenze del giudice costituzionale.

Per quanto concerne, infine, la relazione tra il "diritto vivente" risultante dall'intepretazione giudiziale e l'interpretazione legale fornita dal Tribunale costituzionale, l'esperienza evidenzia come sia assai problematico il rapporto tra il Tribunale supremo -nella sua qualità di organo preposto a realizzare l'unificazione del diritto legale- e il giudice costituzionale -quale garante della conformità del sistema normativo alle disposizioni costituzionali-.

In proposito, può risultare, a nostro avviso, emblematica la considerazione di tre sentenze del Tribunale costituzionale, dalle quali emerge con evidenza la difficoltà di trovare un criterio di comportamento omogeneo nei confronti dell'interpretazione delle disposizioni di legge fornita dalla giurisprudenza del Tribunale supremo. ${ }^{30}$

Nella sentenza 131/06 il Tribunale "salva" la disposizione impugnata, ricorrendo ad una sentenza interpretativa nella quale l'interpretazione conforme è individuata nell'interpretazione fornita dal Tribunale supremo in un precedente ricorso per cassazione (11469/98): secondo il giudice costituzionale l'interpretazione fornita dal Tribunale supremo "es perfectamente asumible para fundar nuestro juicio de constitucionalidad”.

Se nella fattispecie sopra richiamata il Tribunale costituzionale pare far propria la teoria del "diritto vivente", in altre occasioni manifesta, invece, un atteggiamento diverso, orientato a fornire una propria intepretazione della legge difforme

${ }^{29}$ Cfr., V. Crisafulli, Lezioni di diritto costituzionale, cit., 400.

${ }^{30}$ Vedi: J. L. Manzanares Samaniego, La delimitación de competencia entre el Tribunal Supremo y el Tribunal Constitucional, La aplicación jurisdiccional de la Constitución, Valencia, 1997, 63 ss.; M. Del Pilar García RuIz, "El Tribunal supremo como mero órgano unificador de doctrina: la posible afectación de su garantía institucional", in Revista española de derecho constitucional , 2008, 119 ss. 
dall'orientamento del Tribunale supremo. In proposito, merita una breve analisi la STC 212/94 e gli aventi che essa determinato.

Con tale decisione, il Tribunale costituzionale ha risolto un ricorso di amparo presentato contro un Auto della Sala prima del Tribunale supremo il quale -aderendo all' Accordo del Pleno del Tribunale supremo del 20 giugno 1992 circa l'interpretazione da dare al nuovo terzo comma dell'art. 1710 del codice civile in merito ai motivi di inammissibilità dei ricorsi di cassazione civile- aveva ritenuto che nella fattispecie il ricorso poteva essere respinto anche senza " el incidente de audiencia de la parte y la unanimidad en la decisión".

Contro tale applicazione della norma processuale furono presentati diversi ricorsi di amparo costituzionale i quali sostenevano che tale interpretazione costituiva una violazione del diritto di difesa di cui all'art. $24 \mathrm{CE}$, in quanto la decisione avveniva senza la "audiencia de la parte". Il Tribunale costituzionale -nella STC 212/94- analizzò se l'intepretazione dell'art. 1710.3 fornita dal Tribunale costituzionale fosse conforme a Costituzione ed arrivò alla conclusione che "la lectura del precepto revela inequívocamente que en los casos -como el presenteen que la inadmisión del recurso se basa en su falta de fundamento es obligatorio oír al recurrente por el plazo de diez días antes de resolver definitivamente". Di conseguenza, il giudice costituzionale ritenne che l'applicazione della legge da parte del Tribunale supremo si traduceva non tanto in vizio procedurale, quanto in una lesione del diritto di difesa, dal momento che impediva al ricorrente di esporre le proprie ragioni davanti alla Sala del Tribunale supremo.

Siffatta decisione generò (come era prevedibile) forti tensioni tra le due Corti ed il Tribunale supremo sostenne che non poteva essere incrinata la "la libertad del Tribunal Supremo para interpretar con más o menos rigor los requisitos legales de acceso a la casación 'e che non era una' misión del Tribunal Constitucional la interpretación de la legalidad ordinaria”: di conseguenza, stabilì che il decisum del Tribunale costituzionale, in quanto pronunciato in una sentenza conclusiva un ricorso di amparo, non aveva una portata generale, ma inter partes, limitata al caso concreto.

Poichè il Tribunale supremo continuò a risolvere i ricorsi di cassazione sulla base della "sua" interpretazione, furono presentati altri ricorsi di amparo innanzi al Tribunale costituzionale, che si trovò dinanzi ad un'alternativa: o mantenere l'orientamento manifestato nella precedente sentenza oppure tener conto delle argomentazioni fornite dal Tribunale supremo, modificando la propria giurisprudenza. La sua giurisprudenza virò verso quest'ultima soluzione e nel risolvere un ricorso di amparo sostanzialmente identico a quello precedente sostenne che la disposizione di legge offriva diverse interpretazioni conformi a Costituzione e che 
quella adottata dal Tribunale supremo non determinava una lesione del diritto di difesa dal momento che il ricorrente aveva già avuto la possibilità di essere ascoltato al momento della presentazione del recurso de casación (STC 37/95).

Un comportamento ancora differente è stato, infine, tenuto dal giudice costituzionale in occasione della sentenza 63/05, il quale -in materia di prescrizione dei delitti- si differenzia da un orientamento consolidato della giurisprudenza ordinaria. Anche in questo caso la diversità di intepretazione fornita dal Tribunale costituzionale suscita una esplicita reazione del Tribunale supremo, la cui Sala penale ha preso posizione sulla decisione del giudice costituzionale, ribadendo, per un verso, la posizione istituzionale del Tribunale supremo (quale organo giurisdizionale supremo in tutti gli ordini, eccetto in materia di garanzie costituzionali) e confermando, per un altro verso, il proprio orientamento giurisprudenziale "que interpreta que la mera presentación ante el Juzgado de la querella o denuncia basta para producir los efectos interruptivos a los que se refiere el meritado artículo 132.2 del Código Penal”.

Inevitabilmente, quindi, il Tribunale costituzionale fu investito nuovamente della medesima questione; ma in questo caso il giudice costituzionale, a differenza di quanto avvenne in occasione della soprarichiamata sentenza 37/95 ha confermato la propria interpretazione della norma di legge, con conseguente annullamento della sentenza del Tribunale supremo per deficit di motivazione. Nelle motivazioni, il Tribunale precisa che, se è vero che l'applicazione della legislazione ordinaria compete ai giudici e in particolare al Tribunale supremo, tuttavia la loro attività interpretativa incontra un limite quando si verte in materia di garanzie costituzionali, nella quale l'ultima parola spetta al Tribunale costituzionale.

Avverso questa decisione -che si segnala sia perché afferma l'obbligo costituzionale del Tribunale supremo a conformarsi alla doctrina del Tribunale costituzionale, sia in quanto ribadisce la competenza del giudice costituzionale a fornire in materia di diritto processuale penale un'interpretazione della legalità ordinaria differente da quella fornita dal Tribunale supremo- è stato presentato un voto particolare, nel quale si tende a distinguere le due argomentazioni. Nel formularlo, infatti, il giudice Ramón Rodríguez Arribas ritiene, da un lato, che l'istituto della prescrizione sia una materia riservata in via generale alla giurisdizione ordinaria e in particolare alla Sala seconda del Tribunale supremo; dall'altro lato, che la giurisprudenza del Tribunale costituzionale debba, comunque, essere rispettata ai sensi dell'art. 5 della LOPJ.

Le controverse relazioni che intercorrono, in materia di interpretazione legale, tra la giurisprudenza costituzionale e quella del Tribunale supremosono, infine, ulteriormente acuite nei casi in cui i giudici comuni tentano di trovare 
nella giurisprudenza del Tribunale costituzionale un ausilio per far prevalere le loro interpretazioni rispetto a quelle del Tribunale supremo. Non è infrequente, infatti, che questioni di legittimità costituzionale siano sollevate per veder affermata la "validità" di un'interpretazione del diritto legale difforme dalla doctrina del Tribunale supremo.

In proposito, può essere interessante evidenziare come la questione di costituzionalità risolta con la sentenza 38/07 sia stata prospettata dal giudice a quo sulla base di una differente interpretazione dello statuto giuridico dei professori di religione cattolica nelle scuole pubbliche e dell'Intesa tra lo Stato e la Chiesa cattolica del 1979 che aveva contrapposto diversi giudici al Tribunale supremo. In questo -come in altri casi assimilabili- l'oggetto della questione non consisteva tanto nella norma legale, quanto nel fatto che una sua determinata interpretazione potesse ledere un diritto fondamentale: cosicché la questione di costituzionalità finisce per perdere la sua funzione originaria, avvicinandosi a quella propria dei ricorsi diretti a tutela dei diritti fondamentali. ${ }^{31}$

Tuttavia, alla luce degli esempi sopra richiamati, appare evidente il rischio che la competenza del giudice costituzionale a fornire un'interpretazione legale conforme a Costituzione interferisca con il recurso de casación proprio del Tribunale supremo, il quale -secondo la giurisprudenza di quest'ultimo- ha la finalità di revisionare l'applicazione ( sia sostanziale che processuale) della legge da parte dei giudici, al fine di soddisfare i principi di sicurezza giuridica e di eguaglianza nell'applicazione della legge.

Per questa ragione appare condivisibile la prudenza che il Tribunale costituzionale ha manifestato in alcune situazioni al fine di evitare che i giudici comuni possano utilizzare la questione di costituzionalità per veder affermata dal giudice costituzionale un'interpretazione della norma legale differente da quella fornita dal Tribunale supremo. ${ }^{32}$

\section{L'interpretaZione CONFORME A COSTITUZIONE SECONDO LA DOTTRINA DEL TRIBUNALE COSTITUZIONALE}

Il Tribunale costituzionale, in diverse decisioni, ha colto l'occasione sia per precisare le ragioni generali che giustificano il ricorso ad un'interpretazione conforme a Costituzione, sia per indicare le finalità cui tale tecnica interpretativa tende.

\footnotetext{
${ }^{31}$ In proposito, si vedano le osservazioni di: A. GonZÁLEZ Alonso, "Dos oportunidades perdidas. A propósito del control de la constitucionalidad de la designación por parte de la Iglesia de los profesores de religión en la escuela pública”, in Revista general de derecho constitucional, 6. 2008.

${ }^{32}$ Cfr., STC 157/90.
} 
A suo avviso, essa consente di coniugare la supremazia della Costituzione con la conservazione delle disposizioni di legge e rafforza la sicurezza giuridica, in quanto, riconoscendo al sistema normativo una presunzione di legittimità, si evitano i vuoti normativi prodotti da un'eventuale sentenza di incostituzionalità. ${ }^{33}$

Da ciò una naturale propensione ad evitare di dichiarare l' incostituzionalità di una disposizione se può essere interpretata in modo compatibile con la Costituzione. Tuttavia, l'attività interpretativa del giudice costituzionale può far fronte ad uno dei compiti essenziali della giustizia costituzionale -consistente nella "reparación de la incostitucionalidad"- percorrendo itinerari procedurali diversi ed utilizzando tecniche di intepretazione differenti. ${ }^{34}$ In particolare, dinanzi ad una questione di costituzionalità può, anche senza esplicitare i criteri che hanno orientato la sua scelta nel caso concreto, salvare una determinata disposizione ricorrendo ad una sentenza interpretativa ovvero determinarne l'incostituzionalità. Così come, con una certa discrezionalità, può scegliere se inserire l'interpretazione conforme nel dispositivo oppure fare un rinvio generico ai "fondamenti giuridici".

Tuttavia, come evidenzia l'esperienza comparata, la tecnica dell'" interpretazione conforme" può conseguire con maggior efficacia le sue finalità essenziali, per un verso, nei processi per la tutela diretta dei diritti fondamentali piuttosto che in quelli rivolti al controllare la costituzionalità delle leggi; per un altro verso, in quegli ordinamenti che attribuiscono un valore vincolante, erga omnes, all'intera gamma delle sentenze intepretative-come, ad esempio, nel caso della Repubblica federale di Germania e della Spagna-. ${ }^{35}$

Ma il punto più delicato consiste, a nostro avviso, nel rapporto intercorrente tra l'attività interpretativa dei giudici comuni e del giudice costituzionale, dal momento che ogni disposizione è teoricamente suscettibile di una "doppia interpretazione conforme": quella previa (operata dai giudici comuni) e quella successiva (da parte del Tribunale costituzionale). Non va, infatti, dimenticato che -come precisa l'art. 5 della LOPJ- anche i giudici debbono utilizzare le norme costituzionali come criteri per l'intepretazione sistematica dell'ordinamento giuridico

\footnotetext{
${ }^{33}$ Si veda: STC 63/82.

${ }^{34}$ In merito: P. Rodríguez Patrón, La "autonomía procesal" del Tribunal constitucional, Madrid, 2003.

${ }^{35}$ Si veda in proposito: C. STARCK, "Creazione giudiziale del diritto rispetto alla formulazione del dispositivo (Temorierung) e all'efficacia delle sentenze sul controllo di costituzionalità delle norme", in La cricolazione dei modelli e delle tecniche del giudizio di costituzionalità in Europa, Napoli, 2010, 196 ss.; D. SCHEFOLD, L'interpretazione conforme alla Costituzione, ivi, 227 ss.

Meritevole di rinnovato interesse sembra la posizione in passato manifestata da L. EliA, "Sentenze 'interpretative' di norme costituzionali e vincolo dei giudici”, in questa Rivista, 1966, 1697 ss., secondo la quale dovrebbe riconoscersi alla giurisdizione costituzionale la natura di "giurisdizione d'interpretazione", con consecuente vincolo per i giudici sia da parte delle interpretive di accogliemento che di rigetto.
} 
vigente, sino al vincolo di sollevare una questione di legittimità costituzionale solo nel caso in cui risulti impossibile "la acomodación de la norma al ordenamiento constitucional".

Inevitabilmente, quindi, il sistema deve trovare un suo equilibrio attraverso una condivisa ripartizione di compiti tra le due giurisdizioni ${ }^{36}$ ma il conseguimento di tale risultato appare assai difficile, essendo numerose le variabili in gioco. ${ }^{37}$ Ad esempio, dinanzi ad una disposizione suscettibile di esprimere una pluralità di norme il Tribunale costituzionale può scegliere alternativamente se rendere applicabile la norma conforme a Costituzione ovvero se impedire l'utilizzazione dell'interpretazione non conforme; così come, dinanzi ad un'intepretazione del giudice a quo non condivisa, il giudice costituzionale può optare tra il "sollecitare" (con ordinanza) il giudice comune ad utilizzare autonomamente l'interpretazione conforme per risolvere il caso concreto ovvero "costringerlo" (con una sentenza che contiene la doctrina vincolante) a seguire il significato costituzionale corretto individuato dal Tribunale.

Infine, il giudice della costituzionalità delle leggi potrebbe discrezionalmente scegliere se far valere in ogni caso gli effetti normativi previsti dall'art. 40.2 della LOTC secondo cui "la jurisprudencia de los tribunales de justicia recajdas sobre leyes, disposiciones y actos enjuiciados por el TC habrà de entenderse corregida por la doctrina derivata de las sentencias y autos que resulevan los processos constitucionales"; ovvero ricorrere alla forza vincolante della sua doctrina soltanto in situazioni specifiche, come nel caso in cui la ricerca di un significato conforme produca un percorso interpretativo irragionevole, che travalica -cioè- il dato testuale. ${ }^{38}$

${ }^{36}$ Come aveva con chiarezza intuito: P. CAlAmANDREI, "Corte costituzionale e autorità giudiziaria", in Rivista diritto processuale, 1956, 46 allorchè ritiene che per l'efficace esercizio del sindacato di legittimità costituzionale sia necessario che "tra Autorità giudiziaria e Corte costituzionale regni sin da principio buona armonia e identità di criteri”.

${ }^{37}$ Si veda, tra gli altri: S. Ortiz Herrera, Articulación del Tribunal constitucional y del poder judicial en la labor interpretativa de la Constitución. Especial referencia a las sentencias intepretativas del Tribunal constitucional, cit., 633 ss.; R. Punset, J. Santos (dirs.), Jurisdicción ordinaria y Tribunal constitucional, Santiago de Compostela, 1997.

${ }^{38}$ Cfr., STC 16/96. Per una ricostruzione dei profili inerenti ai diversi tipi di sentenze e alla loro utilizzazione da parte del Tribunale costituzionale si rinvia a: F. J. Díaz ReVorio, Las sentencias interpretativas del Tribunal constitucional: significado, tipología, efectos y legitimidad: análisis especial de las sentencias aditivas, Valladolid, 2001; F. Díaz Revorio, "Tipología y efectos de las sentencias de Tribunal constitucional en los procedimientos de inconstitucionalidad ane la reforma de la LOTC", in La Reforma del Tribunal constitucional, cit., 149 ss.; A. Garrorena Morales, "Opacidad y desestimación de la inconstitucionalidad en el fallo de las sentencias intepretativas", in Anuario derecho constitucional y parlamentario, 2000-2001, 137 ss.; H. LÓPEZ BOFILL, Decisiones interpretativas en el control de constitucionalida de la ley, Valencia, 2004; J. Jiménez CAmpo, "Qué 
La semplice enunciazione della molteplicità degli itinerari comportamentali che il Tribunale costituzionale può seguire testimonia l'inevitabilità di una giurisprudenza sul punto non univoca, in molti casi orientata più dalla particolarità del caso concreto che da una meccanica applicazione di un criterio generale. Cionostante non si può sottovalutare il tentativo di darsi alcune regole che fungano da steps argomentativi -da test- cioè da procedimenti logici che il giudice deve necessariamente seguire per attribuire ad una disposizione un determinato significato. Siffatto atteggiamento interpretativo -particolarmente seguito in alcuni ordinamenti di common law-appare pregevole in quanto assicura una certa uniformità di giudizio (dal momento che questioni simili vengono decise sulla base di un medesimo procedimento logico) e rende più trasparente l'iter argomentativo attraverso cui il giudice perviene alla propria ratio decidendi. ${ }^{39}$

Il Tribunale costituzionale non ha, invero, esplicitato in una sentenza i criteri da utilizzare per addivenire ad un'intepretazione conforme; tuttavia, un sistema di regole può essere enucleato dal complesso della sua giurisprudenza, allorchè, nel rispetto del principio di conservazione, giustifica sia una "manipolazione" del testo normativo, sia la possibilità di differenziarsi dalla giurisprudenza dei giudici comuni al fine di stabilire quale sia il significato costituzionale corretto del precetto. ${ }^{40}$

Ad esempio, secondo la giurisprudenza costituzionale: a) se sono possibili due interpretazioni - una conforme e l'altra non conforme a Costituzione- si deve utilizzare la prima; ${ }^{41} \mathrm{~b}$ ) alle sentenze di accoglimento si dovrebbe ricorrere soltanto nelle fattispecie in cui sia "indubbiamente impossibile" pervenire ad un'intepretazione

hacer con la ley inconstitucional”, in Las sentencias sobre la constitucionalidad de la ley, Madrid, 1997, 15 ss.; F. Pera Verdaguer, El uso por el Tribunal constitucional de la t [cnica de las sentencias intepretativas, in La Constitución española, Madrid, 1989, 156 ss.; G. MORA RESTREPO, Justicia constitucional y arbitrariedad de los jueces: teoría de la legitimidad en la argumentación de las sentencias constitucionales, Madrid, 2009.

${ }^{39}$ Sul punto si rinvia a: G. Rolla, Il fascino discreto di una Costituzione, L'apporto della Corte suprema alla determinazione dei caratteri dell'ordinamento costituzionale canadese, Milano, 2008, XIX ss.

${ }^{40}$ Cfr., a titolo di esempio, oltre alle sentenze in seguito citate, STC 83/1983, STC 37/1986. In generale, sull'interpretazione conforme vedi: J. JIMÉNEZ CAMPO, Interpretación conforme, in Enciclopedia juridica básica, Madrid, 1995, 3681 ss.

Per un esame del dibattito generale in materia, si veda, con riferimento alla dottrina in lingua italiana: E. Malfatti, R. Romboli, E. Rossi (cur.), Il giudizio sulle leggi e la sua "diffusione", Torino, 2002; M. D’amico, B. RANDAZZO (cur.), Interpretazione conforme e tecniche interpretative, Torino, 2009; G. SORRENTI, Linterpretazione conforme a Costituzione, Milano, 2006; P. FEMIA (cur.), Intepretazione a fini applicativi e legittimità costituzionale, Napoli, 2006; D. SCHEFOLD, Lintepretazione conforme a Costituzione, La circolazione dei modelli e delle tecniche del giudizio di costituzionalità in Europa, cit., 227 ss.

${ }^{41}$ Cfr., STC 122/83. 
capace di conciliare il significato di una disposizione con i precetti costituzionali; ${ }^{42}$ c) nel caso, poi, di possibilità di una molteplicità di interpretazioni occorre dichiarare solo la non ammissibilità di quella che senza dubbio non appare conforme a Costituzione, lasciando per le rimanenti autonomia interpretativa ai giudici; ${ }^{43}$ d) se sono possibili più interpretazioni egualmente conformi a Costituzione si deve preferire quella "maggiormente conforme": ${ }^{44}$ in questo caso, tuttavia, sarebbe auspicabile che il giudice costituzionale rimanesse sempre fedele all'obiter dictum secondo il quale il Tribunale dovrebbe evitare di scivolare in una sorta di logica ottimizzatrice, che lo spinge anche a selezione tra le possibili quella migliore o "preferibile". ${ }^{45}$ Infine, l'obiettivo della conservazione delle disposizioni non può essere legittimamente perseguito sino al punto di spingersi oltre i confini, le "colonne d'Ercole" costituite dal dato testuale. In altri termini, non è giuridicamente possibile non tener conto del significato degli enunciati legali.

A quest' ultimo proposito, il Tribunale costituzionale ha precisato, in più occasioni, che non è possibile "tratar de reconstruir una norma que no esté debidamente explícita en un texto para concluir que ésta es la norma constitucional"; ${ }^{46}$ che "la efectividad del principio de conservación de las normas (...) no alcanza, como es obvio, a ignorar o desfigurar el sentido de enunciados legales meridianos" (STC 22/85); che "es claro, como consideración de principio, que la interpretación conforme a la Constitución de los preceptos legales tiene también sus límites entre los que se cuenta el del respeto al propio tenor literal de aquéllos". ${ }^{47}$

\section{LA FORZA GIURIDICA DELLA GIURISPRUDENZA DEL}

\section{TRIBUNALE COSTITUZIONALE E LE SENTENZE INTERPRETATIVE}

Il legislatore ha tentato di regolare con apposite disposizioni gli effetti che l'interpretazione del Tribunale costituzionale riverbera nei confronti dell'attività interpretativa dei giudici: sul piano costituzionale, si può segnalare il combinato disposto dell'art. 9.1 CE, che vincola tutti i poteri pubblici al rispetto della Costituzione, e dell'art. dell'art. 161.1 CE, per tutti i pubblici poteri sono soggetti alle decisioni del giudice costituzionale. Così come può essere interessante ricordare

\footnotetext{
${ }^{42}$ Cfr., STC 4/81.

${ }^{43}$ Cfr., STC 93/84.

${ }^{44}$ Cfr., STC $19 / 82$.

${ }^{45}$ Cfr., STC $11 / 81$.

${ }^{46}$ Cfr., STC11/81.

${ }^{47}$ Cfr., STC 222/92.
} 
che il progetto di Costituzione approvato dal Congresso tentava di portare alle estreme conseguenze queste disposizioni generali, disponendo -con specifico riferimento al potere giudiziario- che la giurisprudenza del Tribunale supremo doveva armonizzasi con le pronunce di illegittimità del Tribunale costituzionale. Tale disposizione - non confermata dal Senato durante il procedimento costituente- sembra ricomparire, sia pure in una forma differente prima nella LOTC (1979), quindi nella LOPJ (1985).Tuttavia, le disposizioni presenti nelle due leggi organiche differivano in misura significativa sul punto specifico del carattere vincolante della doctrina del Tribunale costituzionale.

Infatti, l'art. 40.2 del LOTC, nella sua formulazione originaria, affermava che "en todo caso, la jurisprudencia de los Tribunales de justicia recaidas sobre leyes, disposiciones o actos enjuciados por el TC habrá da entenderse corregida por la docrtina derivada de las sentencias y autos que resulevan los recursos y cuestiones de incostitucionalidad". Mentre la successiva LOPJ all'art. 5 imponeva ai giudici di interpretare ed applicare le leggi ed i regolamenti, secondo i precetti ed i principi costituzionali, in conformità_all'interpretazione che risulta dalle decisioni del Tribunale costituzionali dettate "en todo tipo de proceso".

Le due disposizioni coincidevano su un punto rilevante (la natura vincolante della giurisprudenza del Tribunale costituzionale in tema di precetti e principi costituzionali), ma si differenziavano in relazione ad un punto altrettanto importante: infatti, nel caso della LOTC la vincolatività riguardava soltanto le decisioni che risolvono un giudizio di costituzionalità, mentre secondo la LOPJ essa doveva ritenersi estesa alle decisioni inerenti a qualsiasi tipo di processo che si svolge innanzi al Tribunale costituzionale -quindi anche ai giudizi di amparo costituzionale, ai conflitti di competenza, ai conflitti in difesa dell'autonomia locale, ai casi in cui il Tribunale costituzionale pone un'autoquestione di costituzionalità-.

La mancata armonizzazione della normativa -colpisce la constatazione che il legislatore, nonostante abbia revisionato in più occasioni la LOTC (in particolare negli anni 1988, 1999, 2000), non abbia avvertito l'urgenza di allineare il dettato dell'art. 40.2 LOTC all'art. 5 LOPJ- è stata, in passato, fonte incerteza ed ha alimentato situazioni di conflitto, soprattutto per quanto riguardava il valore da attribuire alla doctrina del Tribunale costituzionale nei giudizi relativi ai ricorsi di amparo (se vincolante pro futuro alla luce della LOPJ, ovvero con effetti limitati al caso deciso secondo la LOTC)..$^{48}$

La soprarichiamata discrepanza tra le differenti normative è stata adesso sanata dalla legge organica n. 6/2000, che riformula l'art. 40.2 LOTC, allineandolo alla

${ }^{48}$ In questo senso anche la STC 37/95. 
disciplina contenuta nell'art. 5 LOPJ. L'effetto di questa revisione della legge organica sembra andare oltre ad una mera armonizzazione dei testi legislativi: sia perché, da un lato, si collega ad una differente concezione dell'istituto dell'amparo costituzionale, sia in quanto, dall'altro lato, individua i principi che debbono presiedere alle relazioni tra le due giurisdizioni.

Per quanto concerne il primo profilo, si può notare che un'interpretazione sistematica della LOTC suggerisce una progressiva "obiettivizzazione" di questo istituto. ${ }^{49}$ Secondo l'art. $50.1 \mathrm{~b}$ ) LOTC la possibilità di esperire il ricorso è legata al presupposto che la lamentata lesione di un diritto fondamentale abbia una particolare "trascendencia constitucional": cioè sia importante per l'interpretazione e l'applicazione della Costituzione, per la determinazione del contenuto e della portata dei diritti fondamentali. A sua volta, il Tribunale costituzionale, con un'importante decisione, individuato i casi in cui tale presupposto si determina: quando ha ad oggetto una fattispecie in relazione alla quale non si è ancora formata una doctrina del Tribunale costituzionale; quando il giudice costituzionale avverte la necessità di meglio precisare o di modificare il proprio orientamento giurisprudenziale; quando la lesione di un diritto fondamentale deriva direttamente dal contenuto della legge ovvero da una consolidata intepretazione da parte dei giudici comuni non conforme a Costituzione. Ovvero, quando la giurisprudenza ordinaria è contraddittoria e non dà piena attuazione all'intepretazione costituzionale. ${ }^{50}$

A sua volta, l'art. 40.2 LOTC completa il nuovo disegno costituzionale riconoscendo alle decisioni del Tribunale che risolvono i ricorsi di amparo una portata vincolante per tutti i pubblici poteri. Si ribadisce il principio che la giurisprudenza del Tribunale costituzionale ha la capacità di "correggere" in modo vincolante l'interpretazione dei giudici, costituendo, quindi, una vera e propria fonte del diritto; si precisa che la vincolatività dell'interpretazione fornita dal Tribunale costituzionale si riferisce alla dottrina costituzionale formulata in tipi di processi e risultante da tutti i tipi di sentenze - da quelle di accoglimento a quelle interpretative-.

$\mathrm{Va}$, infine, considerato che tale dottrina si forma non solo sulla base del dispositivo, ma anche delle rationes decidendi: cosicché l'effetto vincolante si

\footnotetext{
${ }^{49}$ Per una aggiornata ricostruzione del dibattito in ordine alle "nuova natura" del ricorso di amparo si rinvia al lavoro di R. Tur Ausina, Garantía de derechos y jurisdicción constitucional, cit., 2008. Una valutazione complessiva della reforma della LOTC è stata operata, di recente, da: S. GARCíA VÁZQUEZ, La controvertida reforma de la Ley Orgánica del Tribunal constitucional, Santiago de Compostela, 2009.

${ }^{50}$ Così in STC 155/09.
} 
estende alle motivazioni che supportano la decisione, consentendo al Tribunale costituzionale di imporre meglio le "proprie concezioni giuridiche in materia di diritto costituzionale". ${ }^{51}$

La forza vincolante delle decisioni del Tribunale costituzionale si manifesta, tuttavia, con profili distinti a seconda del tipo di competenza esercitata.

Le sentenze che concludono un ricorso di amparo contro atti o omissioni del potere giudiziario vincolano il giudice che deve rimediare alla lesione di un diritto fondamentale, ma condizionano, pro futuro, anche l'autonomia interpretativa dei giudici, i quali debbono interpretare ed applicare le leggi ed i regolamenti in conformità all'interpretazione che risulta dalle decisioni del Tribunale costituzionali dettate "en todo tipo de proceso". Tuttavia, il valore vincolante della doctrina costituzionale non è indiscriminato, nel senso che -come emerge dall' Auto 232/92 del Tribunale costituzionale- vale solo nel caso che anche in futuro si producano lesioni della stessa disposizione costituzionale in situazioni successive aventi la "misma relación jurídica".

Invece, le sentenze che risolvono una questione di incostituzionalità sono suscettibili di produrre effetti differenziati a seconda della tecnica decisoria utilizzata dal giudice costituzionale..$^{52}$

La forza vincolante delle decisioni del Tribunale costituzionale non pone problemi particolari quando esso dichiara l'incostituzionalità di una disposizione, dal momento che l'art. 39.1 LOTC precisa che "cuando la sentencia declare la incostitucionalidad, declarará igualmente la nulidad de los preceptos impugnados". Tuttavia, il nesso consequenziale "incostituzionalità-nullità delle disposizioni” richiamato dal legislatore organico presuppone un Tribunale costituzionale che opera nel sistema come mero "legislatore negativo": un'immagine che-come è noto- non corrisponde all'evoluzione delle esperienze di giustizia costituzionale, ove con grande frequenza non si dichiara tanto "la nulidad de los preceptos impugnados", quanto la conformità o non conformità alla Costituzione delle norme che scaturiscono dall'intepretazione. $\mathrm{O}$, come meglio è stato precisato da un'autorevole dottrina, il giudice costituzionale denuncia

\footnotetext{
${ }^{51}$ Per ulteriori considerazioni in ordine agli effetti delle decisioni del Tribunale costituzionale si veda: R. Bocanegra Sierra, El valor de las sentencias del Tribunal constitucional, Madrid, 1982; G. Rolla, Indirizzo politico e Tribunale costituzionale in Spagna, cit., 278 ss.

52 Si vedano: M. C. Blasco Soto, La sentencia en la cuestión de inconstitucionalidad, Barcelona, 1995; J. JimÉNEZ CAMPO, "Qué hacer con la ley inconstitucional", in Las sentencias sobre la constitucionalidad de la ley, cit., 15 ss.; F. J. Díaz ReVorio, Las sentencias interpretativas del Tribunal constitucional: significado, tipología, efectos y legitimidad: análisis especial de las sentencias aditivas, cit., 2001.
} 
-attraverso il richiamo formale della disposizione scritta- una determinata norma da essa deducibile per via di interpretazione..$^{53}$

E' il caso, ad esempio, delle sentenze "integradoras", che si hanno allorchè l'intepretazione contempla una necessaria attività di integrazione normativa -specialmente quando il parametro del giudizio di costituzionalità è costituito dal principio di eguaglianza e dal divieto di discriminazione- al fine di colmare una lacuna, la cui persistenza determina una lesione della Costituzione. In proposito, il Tribunale costituzionale ha riconosciuto che il compito di interpretare le disposizioni di legge in conformità con i principi e i diritti costituzionali richiede non solo una "interpretación declarativa de las normas jurídicas, sino también una "interpretación integradora", cuando así lo imponga la adecuación de la norma a la Constitución", ${ }^{54}$ ovvero la necessità di sanare la carenza di una precetto legale con l'applicazione diretta di un precetto costituzionale. ${ }^{55}$

Così come si possono richiamare le sentenze di "incostituzionalità parziale qualitativa", che si hanno -ad avviso della dottrina- nei casi in cui l'incostituzionalità è prodotta non dal testo normativo, ma in seguito all'applicazione della legge in determinate situazioni. In questo caso, il Tribunale costituzionale evita di annullare la disposizione, ma modifica le condizioni della sua applicazione: in tal modo, il giudice costituzionale provoca una modificazione dell'ambito di applicazione della legge introducendo precisazioni addizionali (che non compaiono nell'enunciato normativo), le quali possono riguardare sia i destinatari, che i presupposti per la sua applicazione o le circostanze in cui può essere legittimamente applicata. ${ }^{56}$

Un sottotipo di queste sentenze è costituito, poi, dalle sentenze "reductoras", che si limitano a ridurre l'ambito di applicazione degli enunciati legali. E' il caso, ad esempio, della STC 87/91 che ha dichiarato l'incostituzionalità di una disposizione "per quanto riguarda il computo del tempo di prescrizione"; ovvero della STC 5/81 che ha dichiarato l'incostituzionalità di una norma "in quanto si riferisce a centri sostenuti dall'amministrazione pubblica con fondi pubblici, mentre non è contraria a Costituzione nel caso di centri privati non sostenuti con fondi pubblici”.

Tuttavia, nelle ipotesi soprarichiamate, il particolare tipo di tecnica decisoria utilizzato dal Tribunale costituzionale crea, comunque, un vincolo per i giudici

\footnotetext{
${ }^{53}$ Si veda, V. CRISAFulli, "Questioni in tema di interpretazione costituzionale nei rapporti con l'interpretazione giudiziaria”, in questa Revista, 1956, 939.

${ }^{54}$ Cfr., STC 103/90.

${ }^{55}$ Cfr., STC 74/87.

56 Vedi: M. Ahumada, "El control de constitucionalidad de las omisiones legislativas", in Revista Centro estudios constitucionales, 1991, 169 ss.
} 
-al pari di tutti gli operatori del diritto-, dal momento che la possibilità per una disposizione di continuare a produrre effetti giuridici è strettamente condizionata dall'attribuzione alla stessa del significato "creativo" attribuitole dal Tribunale e rinvenibile -normalmente- nel "considerato in diritto" della sentenza.

Più complesse sono le relazioni tra le due giurisdizioni nel caso delle sentenze "interpretative", cioè di quelle decisioni che rigettano una questione di incostituzionalità qualora si interpreti la disposizione nel modo che il Tribunale costituzionale considera adeguato alla Costituzione ovvero non lo si interpreti nel senso ritenuto non conforme a Costituzione. Quindi il suo carattere interpretativo si manifesta sia quando il Tribunale costituzionale indica l'intepretazione conforme, sia nelle fattispecie in cui esclude una possibile interpretazione. ${ }^{57}$

Le sentenze di tipo interpretativo possiedono una duplice caratteristica.

Innanzitutto, l'esito del processo è, comunque, necessariamente "desestimatorio", nel senso che il Tribunale costituzionale dichiara nel dispositivo la costituzionalità della disposizione "si se interpreta" o "interpretada" conformemente a quanto indicato nel dispositivo nella ratio decidendi della sentenza. In secondo luogo, l'interpretazione è parte integrante della decisione e la stessa interpretazione -stante il suo rapporto di stretta conseguenzialità con il decisum- costituisce un vincolo per i giudici comuni alla luce dell'art. 40.2 LOTC e dell'art. 5 LOPJ.

Si tratta -riprendendo le parole del giudice costituzionale- di "un medio licito", ma "de muy delicato y difícil uso", cui è opportuno far ricorso con una certa cautela; ${ }^{58}$ inoltre, dal momento che l'intepretazione conforme del Tribunale costituzionale produce una doctrina vincolante in grado di correggere differenti orientamenti intepretativi dei giudici comuni, sarebbe opportuno precisare e rendere evidente (senza lasciare dubbi o incertezze) il significato dell'intepretazione della norma legale fornita dal giudice costituzionale. Si dovrebbe, in altri termini, estendere al Tribunale costituzionale l'esigenza, richiesta al legislatore, di perseguire la chiarezza e non la confusione normativa in modo che gli operatori giuridici sappiano a che cosa si debbono attenere. ${ }^{59}$

A questo proposito, ha generato perplessità la tendenza ad adottare sentenze il cui dispositivo è formalmente di rigetto, mentre gli effetti interpretativi debbono

\footnotetext{
${ }^{57}$ Tra l'ampia dottrina che ha approfondito i caratteri delle sentenze intepretative, si veda: F. DíAz Revorio, Tipología y efectos de las sentencias de Tribunal constitucional en los procedimientos de incostitucionalidad ante la reforma de la LOTC, cit., 149 ss.; H. LOPEZ BOFILL, Decisiones interpretativas en el control de constitucionalidad de la ley, cit., 2004; F. Pera Verdaguer, El uso por el Tribunal constitucional de la técnica de las sentencias intepretativas, cit., 156 ss.

${ }^{58}$ Cfr., STC $4 / 81$.

${ }^{59}$ STC $46 / 90$.
} 
essere ricercati nel corpo del "considerato in diritto": 60 si è parlato, in proposito, di "sentencias interpretativas sin reflejo en el fallo". ${ }^{61}$ Non sono mancate, inoltre, decisioni in occasioni delle quali la maggioranza dei giudici costituzionali ha ritenuto che nel caso concreto le disposizioni possono "intepretarse de modo natural y no forzado, y sin afectación de la securidad jurídica” nel modo indicato dal Tribunale nella "parte in diritto". ${ }^{62}$

Il ricorso a tale tipo di sentenze è stato contestato in dottrina con la considerazione che non hanno un dispositivo univoco e, rinviando genericamente ai "fundamentos jurídicos", si pongono ai margini del principio di sicurezza giuridica. ${ }^{63}$ Anche all'interno del collegio non sono mancate posizioni critiche, manifestate in occasione di voti particolari. Ad esempio, il giudice Tomás y Valiente aveva evidenziato in un suo voto particolare alla STC 5/1981 la necessità per il Tribunale costituzionale di "llevar al fallo la intepretación de la norma impugnada" al fine di ridurre le ambiguità della legge e di promuovere la sicurezza giuridica. Mentre, a sua volta, il giudice Jiménez de Parga ha sostenuto nel voto particolare alla STC n.176/99 che le differenti, contradditorie interpretazioni della disposizione impugnata rendono necessario un pronunciamento espresso, cioè un "fallo interpretativo" e non un mero riferimento ai fondamenti giuridici.

\section{L'INTERPRETAZIONE CONFORME A COSTITUZIONE NEI RICORSI DI AMPARO COSTITUZIONALE}

Sin dai primi anni di funzionamento della giustizia costituzionale, apparve chiaro non solo che la competenza in materia di ricorsi di amparo avrebbe assorbito l'attività del Tribunale costituzionale, ma anche che la maggior parte degli stessi ricorsi avrebbe riguardato atti od omissioni dei giudici nell'esercizio della loro funzione giurisdizionale. ${ }^{64}$

Tale fenomeno, oramai consolidato, pone dei problemi non secondari nelle relazioni tra le due giurisdizioni, qualora si consideri che l'istituto dell'amparo

\footnotetext{
${ }^{60}$ Si possono richiamare, a titolo esemplificativo: STC 64/82; STC 71/82, STC 76/83, STC 176/99, STC $74 / 00$.

${ }^{61}$ Così, ad esempio: A. Garrorena Morales, Opacidad y desestimación de la incostitucionalidad en el fallo de las sentencias interpretativas, cit., 137 ss.

${ }^{62}$ Cfr., STC 167/99, STC 74/00.

${ }^{63}$ Tale critica è stata avanzata da: J. SANTOS VIJANDE, Doctrina y jurisprudencia del Tribunal constitucional. Su eficacia y respecto de los tribunales ordinarios, Granada, 1995; A. Garrorena Morales, Opacidad y desestimación de la inconstitucionalidad en el fallo de las sentencias interpretativas, cit., 137 ss.

${ }^{64}$ Siffatta eventualità era già stata ipotizzata in: G. Rolla, Indirizzo politico e Tribunale costituzionale in Spagna, cit., 264.
} 
costituzionale, da un lato, ammette un controllo sull'attività giudicante dei giudici da parte di un potere estraneo all'ordine giudiziario, dall'altro lato, riconosce alle sentenze del Tribunale costituzionale il potere non solo di modificare la decisione del giudice (avverso la quale è stato presentato il ricorso), ma anche di modificare pro futuro la giurisprudenza alla luce della doctrina del Tribunale costituzionale espressa a conclusione del giudizio.

La stessa genesi di questa competenza conteneva i germi di un possibile, continuo "conflitto" tra le giurisdizioni: d'altra parte, ad avviso di settori autorevoli della dottrina, l'istituzione di tale ricorso sarebbe non solo il risultato di un fisiologico processo di circolazione giuridica ( nel nostro caso, l'amparo americano e il ricorso diretto a tutela dei diritti fondamentali della Repubblica federale tedesca), non solo un tributo alla tradizione storica (nello specifico, la breve esperienza costituzionale della Seconda Repubblica), ma anche la manifestazione di un atteggiamento dei costituenti di "cautela" nei confronti dei giudici, motivato dalla convinzione che essi non sarebbero stati in grado di interiorizzare con rapidità i nuovi valori costituzionali in materia di diritti. Da ciò anche la scelta di individuare nel Tribunale costituzionale l'organo supremo cui affidare, in materia di diritti fondamentali, la formazione di un orientamento giurisprudenziale vincolante per tutti i poteri dello Stato.

Non stupisce, quindi, se tale competenza fu subito contestata: sia all'interno dell'ordine giudiziario, da parte di chi riteneva che il Tribunale costituzionale non avesse le caratteristiche proprie del giudice naturale predeterminato per legge; sia da parte della dottrina, la quale evidenziava criticamente come il giudice costituzionale fosse, in tal modo, abilitato a rettificare "el poder de apreciación judicial, contrastando y ponderando la discrecionalidad judicial". ${ }^{65}$

Alle iniziali perplessità si aggiunsero -a mano a mano che si sviluppava la giurisprudenza del Tribunale costituzionale- altre cause di tensione tra le due giurisdizioni: alcune di queste riconducibili all'evoluzione (o meglio, progressiva trasfigurazione) dell'istituto, altre in conseguenza dell'ampliamento delle posizioni soggettive tutelabili in sede di amparo.

Tra le prime può essere annoverata l'affermarsi di una certa tendenza a "sviare" l'istituto dell'amparo dal suo alveo naturale, nel senso che in diversi casi è sembrato che l'obiettivo primario della decisione non fosse tanto la protezione di un diritto fondamentale, quanto la correzione di un orientamento giurisprudenziale che, in

\footnotetext{
${ }^{65}$ Cfr., L. Martín Retortillo, Del control de la discrecionalidad administrativa al control de la discrecionalidad judicial, cit., 1088 ss.; I. Dírz Picazo, "Reflexiones sobre el contenido y efectos de las sentencias dictadas por el Tribunal constitucional en recursos de amparo", in La sentencia de amparo constitucional, Madrid, 1996, 72 ss.
} 
quanto non conforme a Costituzione, avrebbe prodotto la lesione di un diritto. In altri termini, il Tribunale costituzionale è tentato talvolta di utilizzare l'istituto per compiere un'attività interpretativa più consona al giudizio di costituzionalità in via incidentale che a quello di amparo. ${ }^{66}$

Emblematica appare, in proposito, la vicenda che ha portato alla STC 7/94.

Alla base del ricorso vi era una domanda di riconoscimento di paternità presentata innanzi ad un giudice di prima istanza, il quale la rigettò in quanto il convenuto si era rifiutato di sottoporsi ad un test di paternità. Il giudice di seconda istanza, dinanzi al reiterarsi del rifiuto, considerò lo stesso un indizio sufficiente per riconoscere la paternità del convenuto, una sorta di ficta confessio. Il Tribunale supremo fu -a sua volta- di differente avviso, non considerando il rifiuto di sottomettersi al test di paternità una confessione e ritenendo, d'altra parte, che non sussistevano altri elementi che potessero far ritenere che il convenuto fosse il padre.

Il Tribunale costituzionale, nel corso del giudizio di amparo, affermò che il test di paternità è una prova decisiva per determinare la paternità e che il rifiuto ad eseguirlo privava la ricorrente di un elemento decisivo su cui fondare la sua difesa, in violazione dell'art. $24 \mathrm{CE}$. Pertanto, il Tribunale costituzionale -con una sentenza che ha suscitato vibrate proteste da parte del Tribunale supremo- ha annullato la decisione del Tribunale supremo, ripristinando la sentenza del giudice di seconda istanza.

Un altro profilo problematico evidenziato dalla prassi seguita dal giudice costituzionale è rappresentato dalla possibilità per il Tribunale costituzionale di utilizzare un processo di amparo costituzionale non solo per rimediare in un caso specifico alla lesione di un diritto fondamentale, ma anche per espungere dall'ordinamento con effetti generali le disposizioni di legge ritenute contrarie alle norme costituzionali in materia di diritti fondamentali.

Dapprima, in via di prassi, il giudice costituzionale ha iniziato a sollevare, nel corso di un giudizio di amparo, un' "autoquestione di costituzionalità", con l'evidente finalità di attribuire effetti generali all'interpretazione conforme a Costituzione sulla base della quale ha risolto il ricorso. Emblematica è, in proposito, la STC 472/00 nella quale il Tribunale costituzionale, nell'annunciare l'accoglimento dell' amparo e la contestuale prospettazione di un' "autoquestione" di incostituzionalità, motivava tale scelta nei seguenti termini: "como hemos

${ }^{66}$ E' il caso, ad esempio, delle STC 111/93 e 150/97.

Per ulteriori riflessioni in merito si rinvia a: P. Pérez Tremps, Tribunal Constitucional y Poder judicial, cit., 1985. 
destacado en el fundamento jurídico 5, la Ley aplicada vulnera el art. 17 C.E. y esa vulneración ha podido ser determinante de la actuación inconstitucional de los órganos judiciales, por lo que se está en el supuesto previsto en el art. 55.2 LOTC y procede, por tanto, plantearse la cuestión de inconstitucionalidad relativa a dichos preceptos".

Successivamente, la legge organica n. 6/2007 ha codificato tale comportamento prevedendo, nella nuova formulazione dell'art. 52.2 LOTC, che qualora il Tribunale costituzionale ritenga che un ricorso debba essere accolto in quanto la legge applicata viola un diritto fondamentale, si deve sospendere il giudizio in merito all'amparo, sollevando la questione dinanzi al pleno del Tribunale.

Le difficoltà nel rapporto tra giurisdizione costituzionale e ordinaria sono state alimentate anche dall'intepretazione estensiva data ai diritti di difesa e ad una tutela giudiziale effettiva garantiti dall'art. $24 \mathrm{CE} .{ }^{67}$

Il giudice costituzionale, invero, ha compiuto alcuni tentativi per circoscrivere la portata del proprio sindacato: ad esempio, ha introdotto una distinzione tra "indefensión procesal" e "indefensión constitucionalmente relevante", così come ha riconosciuto che la discrezionalità dei giudici comuni nell'esame delle fattispecie concrete è un elemento indissociabile dall'esercizio della funzione giurisdizionale. ${ }^{68}$ Inoltre, in diverse occasioni, ha precisato che il ricorso di amparo non può divenire una terza istanza giurisdizionale, trasformando il giudice costituzionale un organo "censor" del potere giudiziario; oppure ha riconosciuto che il giudizio trova un limite nella valutazione dei fatti e degli elementi di prova o nell'apprezzamento degli elementi di giudizio valutati dal giudice. ${ }^{69}$

Tuttavia, a causa della natura di questa competenza e della formulazione del diritto alla tutela giudiziale effettiva contenuta nell'art. $24 \mathrm{CE}$, il Tribunale costituzionale si è trovato più volte nella condizione di controllare l'interpretazione della legalità ordinaria fornita dai giudici, in quanto considerata un'operazione necessaria, un presupposto per valutare se si sia prodotta o no una violazione di tale diritto fondamentale.

D'altra parte, anche se non tutto il diritto processuale è stato costituzionalizzato in virtù dell'art. $24 \mathrm{CE}$, è altrettanto indubbio che il Tribunale costituzionale-cui compete per legge delimitare il proprio ambito di giurisdizione (art. 4 LOTC)-

\footnotetext{
${ }^{67}$ In proposito, si veda: I. Borrajo - I. Díez Picazo - G. Fernándes Farreres, El derecho a la tutela judicial y el recurso de amparo, Madrid, 1995; I. BORRAJO INIESTA, El derecho a la tutela judicial y el recurso de amparo: una reflexión sobre la jurisprudencia constitucional, Madrid, 1995.

${ }^{68}$ Cfr., STC 8/81.

${ }^{69}$ Cfr., STC 41/84 , STC 329/88.
} 
rimane il dominus nel distinguere quali garanzie abbiano una base legale e quali costituzionale. E' evidente che il giudice costituzionale, nel mentre amplia il novero delle garanzie processuali riconducibili all'art. $24 \mathrm{CE}$, accresce la sua capacità di sindacare l'applicazione da parte dei giudici di norme legali; così come, attraverso il richiamo al diritto ad una tutela giudiziale effettiva, finisce per sottoporre a revisione critica i comportamenti processuali, nonché l'iter argomentativo seguito dal giudice.

In particolare, alcuni profili della sua giurisprudenza appaiono meritevoli di attenzione, in quanto evidenziano la difficoltà di tenere distinti gli ambiti di competenza delle due giurisdizioni.

Innanzitutto, il sindacato del giudice costituzionale non comprende solo gli errori in diritto, ma si estende anche a sindacare gli errori di fatto compiuti dai giudici nel corso di un processo. Rientrano in questa ultima fattispecie i casi in cui la lesione dell'art. $24 \mathrm{CE}$ è determinata da un errore manifesto - "error patente" secondo la terminologia utilizzata dal Tribunale-, da un incontrovertibile errore nella ricostruzione dei fatti. In caso di errori nell'intepretazione e nell'applicazione delle norme -sia sostanziali che processuali- il giudice costituzionale ha utilizzato, poi, l'intera gamma dei vizi propri della discrezionalità: dal principio di proporzionalità, ${ }^{70}$ al criterio di ragionevolezza, alla regola secondo cui tutte le scelte debbono fondarsi su criteri oggettivi. ${ }^{71}$

Secondo il Tribunale costituzionale un diritto fondamentale può essere leso anche da una motivazione del tutto inadeguata, ${ }^{72} \mathrm{da}$ un comportamento processuale incongruente, ${ }^{73}$ dalla mancanza di una razionalità interna all'atto del giudice, ${ }^{74}$ dall'assenza di una corrispondenza sostanziale tra le domande avanzate dalle parti e le risposte fornite dall'autorità giurisdizionale: a quest'ultimo proposito, il Tribunale costituzionale ha parlato di "incongruenza tale da determinare una completa modificazione dei termini del dibattito processuale". ${ }^{75}$

Così come, basandosi sulla considerazione che il diritto ad una tutela giudiziale effettiva comprende anche il diritto ad ottenere una decisione giudiziale fondata su norme giuridiche, ha ammesso la possibilità di verificare la sussistenza di una motivazione non arbitraria. Con la STC 55/93 il Tribunale costituzionale si ritiene competente ad esaminare i motivi e gli argomenti sui quali si fonda la

\footnotetext{
${ }^{70}$ Cfr., STC 30/81.

${ }^{71}$ Cfr., STC 30/82.

${ }^{72}$ Cfr., STC 46/82.

${ }^{73}$ Cfr., STC 255/83.

${ }^{74}$ Cfr., STC 14/84.

${ }^{75}$ Cfr., STC 10/81.
} 
decisione giudiziale impugnata, al fine di valutare se sono ragionevoli nella prospettiva costituzionale, in modo che con l'amparo costituzionale si possa correggere un'interpretazione arbitraria o che evidenzi un errore chiaro con rilevanza costituzionale. ${ }^{76}$ Inoltre, il Tribunale ha ritenuto che ledesse l'art. $24 \mathrm{CE}$ una sentenza la quale contenga contraddizioni interne ed errori tanto evidenti da dar vita ad una decisione irrazionale ${ }^{77}$ così come ha ritenuto che lesione di un diritto possa derivare anche da un'applicazione erronea di una disposizione adeguata ovvero dalla risoluzione di una questione sulla base di una norma che non si sarebbe dovuto utilizzare. ${ }^{78}$

Secondo la giurisprudenza costituzionale in materia di amparo il diritto costituzionale ad una tutela giudiziale effettiva sarebbe violato anche in caso di "dilazioni indebite" da parte del giudice, per cui il Tribunale si riconosce competente a sindacare atti o omissioni di natura procedurale, suscettibili di provocare un' irragionevole durata del processo.

La giurisprudenza del Tribunale costituzionale si è focalizzata anche sui comportamenti di natura processuale suscettibili di ledere il diritto di difesa.

In alcune occasioni, ha ammesso, specie in relazione al principio di presunzione di innocenza, la possibilità di verificare se il giudice ha compiuto un'attività "suficiente de aportación de pruebas": cioè, se effettivamente in quel caso si sono prodotte delle prove. Interessante è anche la STC 119/1993, ove si afferma che l'interpretazione e l'applicazione della legalità ordinaria può essere oggetto di un amparo costituzionale quando leda direttamente alcuni diritti processuali. Sempre nella medesima sentenza si precisa che gli eventuali errori che i giudici hanno commesso nel risolvere questioni di mera legalità possono divenire oggetto di valutazione da parte del Tribunale costituzionale qualora essi impediscano -ad esempio- la possibilità di presentare un ricorso, l'impossibilità di esercitare il diritto di difesa.

Alla luce degli orientamenti giurisprudenziali sommariamente richiamati non può sfuggire che alcuni dei vizi richiamati per risolvere i ricorsi di amparo (errore evidente, arbitraria applicazione della legge, irragionevole argomentazione) sono gli stessi che costituiscono il fondamento della competenza del Tribunale supremo per riconoscere un indennizzo per errore giudiziale. Si lamenta da alcune parti, quindi, che il Tribunale costituzionale abbia aperto una seconda e parallela via processuale per riparare gli errori giudiziali: affiancando alla competenza del Tribunale supremo, il ricorso costituzionale.

${ }^{76}$ Cfr., STC 23/87, STC 201/87, STC 63/90.

77 Cfr., STC 175/96.

${ }^{78}$ Cfr., STC 304/82. 
